INPLASY

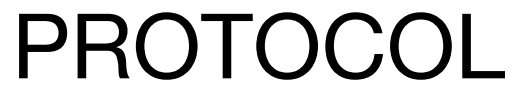

To cite: Rong et al.

Levonorgestrel-releasing intrauterine system versus medications for women with heavy menstrual bleeding: A systematic review and metaanalysis. Inplasy protocol

202050095. doi:

10.37766/inplasy2020.5.0095

Received: 26 May 2020

Published: 26 May 2020

Corresponding author:

Lan Zhu

florafr@163.com

Author Affiliation:

Peking Union Medical College

Hospital (PUMCH)

Support: None

Review Stage at time of this

submission: Preliminary

searches.

Conflicts of interest:

None.

\section{Levonorgestrel-releasing intrauterine system versus medications for women with heavy menstrual bleeding: A systematic review and meta-analysis}

Rong, F; Ke, D²; Ling, L3; Jing, W4; Lan, Z5.

Review question / Objective: To compare the efficacy and safety of levonorgestrel-releasing intrauterine system versus medications for women with heavy menstrual bleeding.

Condition being studied: Females (age $\geq 18$ years) with heavy menstrual bleeding.

Information sources: We will search the electronic databases (including PubMed, Embase, the Cochrane Liabrary, CNKI, and Wanfang), without any limitation on specific languages, regions or publication year.

INPLASY registration number: This protocol was registered with the International Platform of Registered Systematic Review and Meta-Analysis Protocols (INPLASY) on 26 May 2020 and was last updated on 26 May 2020 (registration number INPLASY202050095).

\section{INTRODUCTION}

Review question / Objective: To compare the efficacy and safety of levonorgestrelreleasing intrauterine system versus medications for women with heavy menstrual bleeding.
Condition being studied: Females (age $\geq 18$ years) with heavy menstrual bleeding.

\section{METHODS}

Participant or population: We will include women (age $\geq 18$ years) that was diagnosed of heavy menstrual bleeding. Heavy menstrual bleeding is generally 
defined as blood loss of $80 \mathrm{ml}$ or more or a condition when the duration of menses exceeds 7 days.

Intervention: Levonorgestrel-releasing intrauterine system.

Comparator: Medications without any limitation on type, dosage regimens or treatment duration.

Study designs to be included: All randomized controlled trials (RCTs) will be included.

Eligibility criteria: All randomized controlled trials investigated levonorgestrel-releasing intrauterine system versus medications for women with heavy menstrual bleeding. Language in English or Chinese.

Information sources: We will search the electronic databases (including PubMed, Embase, the Cochrane Liabrary, CNKI, and Wanfang), without any limitation on specific languages, regions or publication year.

Main outcome(s): 1. Clinical response to treatment (as defined in individual trials); 2. Menstrual blood loss (measured by pictorial blood loss assessment chart (PBAC), Menorrhagia Multi-Attribute Scale (MMAS), or other valid ways); 3. Quality of life measured by any validated scale (such as Health Related Quality of Life (HRQoL-4)). We will collect the outcome data measured at different timepoints reported in each study.

Additional outcome(s): 1. Patients satisfaction; 2. Amenorrhea; 3. Basic methemoglobin; 4. Haemoglobin (Hb); 5. Withdrawal of Treatment; 6. Number of drop-out; 7. Adverse events (AEs). We will collect the outcome data measured at different timepoints reported in each study.

Data management: We will design a standard data extraction form. The following information will be extracted by two reviewers independently: 1 . Identification of the study (study ID; first author; country of publication and geographical setting of the study; number of centers; trial sponsors; and year of study); 2. Information relevant to study design (the randomization method; allocation concealment; blinding method); 3. Participants (age; study inclusion and exclusion criteria; total participant number in each treatment group, baseline characteristics including menstrual characteristics, body mass index, burst size); 4. Interventions (specific type, dosage and frequency; treatment duration); 5. Outcomes (pre-specified primary and secondary outcomes in the protocol; other outcomes reported by each study; dropouts; follow-up; results data). Any disagreements will be resolved by discussion, with the assistance from a third party if necessary.

Quality assessment / Risk of bias analysis: Two reviewers will assess the quality of included studies independently. We will evaluate every domain of risk of bias by the Cochrane tool for RCT (Higgins 2011), including sequence generation, allocation concealment, blinding of participants and personnel, blinding of outcome assessment, incomplete outcome data, selective outcome reporting, and other bias. We will generate a 'Risk of bias graph' and a 'Risk of bias' summary'. 'Low risk' will be shown in green, 'unclear risk' will be shown in yellow and 'high risk' will be shown in red. Disagreements were resolved by discussion, with the assistance from a third party if necessary.

Strategy of data synthesis: We plan to summarize all dichotomous outcome data using risk ratio (RR) and its $95 \%$ confidence interval (Cl), and to summarize all continuous outcome data using mean difference (MD) and its $95 \%$ confidence interval (Cl). We will consider and fully discuss the clinical and methodological heterogeneity, and make the priori subgroup analysis based on potential factors (See Subgroup analysis). Significant statistical heterogeneity is evaluated by chi-square test $(P<0.1)$ and $I^{2}$ statistics $(\geq$ $50 \%$ ). We will assess the reporting bias through visual interpretation of funnel plots and Egger's test for the outcomes included 10 or more than studies. We will use 
RevMan 5.3 (RevMan 2014) to conduct meta-analysis. We understand that there is no closed argument for preference for use of fixed-effect or random-effects models. We will choose to use random-effects model for all meta-analysis. If metaanalysis is considered inappropriate, we will describe the outcome data separately.

Subgroup analysis: We plan to perform subgroup analysis on primary outcomes according to type of medications (nonsteroidal versus steroidal).

Sensibility analysis: We plan to perform sensitivity analysis on clinical response according to criteria of which.

Country(ies) involved: China.

Keywords: levonorgestrel-releasing intrauterine system; heavy menstrual bleeding.

Contributions of each author:

Author 1 - Rong Fan - Rong Fan drafted the manuscript, review the manuscript.

Author 2 - Ke Deng - Ke Deng drafted the manuscript.

Author 3 - Ling Li - Ling Li drafted the manuscript.

Author 4 - Jing Wang - Jing Wang drafted the manuscript.

Author 5 - Lan Zhu - Lan Zhu drafted the manuscript, review the manuscript. 